\title{
Comprehensive Genomic Profiling for Therapeutic Decision and Identification of Gene Mutation in Uterine Endometrial Dedifferentiated Carcinoma
}

\author{
Yusuke Taira Yuko Shimoji Yoshihisa Arakaki Tomoko Nakamoto \\ Wataru Kudaka Yoichi Aoki \\ Department of Obstetrics and Gynecology, Graduate School of Medicine, University of the \\ Ryukyus, Okinawa, Japan
}

\section{Keywords}

Endometrial dedifferentiated carcinoma · Comprehensive genomic profiling · Gene mutation - Therapeutic target

\section{Abstract}

Endometrial dedifferentiated carcinoma is a new concept among endometrial malignancies, is rare, and has a poor prognosis as it is discovered in advanced stages and has no established treatment. It has higher rates of gene mutations, such as mismatch repair, than general endometrial cancer and has been associated with Lynch syndrome. However, due to its heterogeneity, case-by-case searches are needed. Comprehensive genomic profiling by Foundation One ${ }^{\circledR}$ CDx can comprehensively identify over 300 gene mutations via a next-generation sequencer and can evaluate biomarkers, such as the microsatellite status and tumor mutation burden. Therefore, it may be beneficial in identifying therapeutic targets and drugs for diseases whose treatment has not been established. In this case, 13 therapies, including immune checkpoint inhibitor therapy for microsatellite instability-High and 40 clinical trials for several gene mutations might be beneficial. We report a case of endometrial dedifferentiated carcinoma for which Foundation One ${ }^{\circledR} C D x$ gene profiling was used to propose treatment.

\section{Karger ${ }^{\prime \prime}=$}




\section{Introduction}

Dedifferentiated carcinoma of the uterine corpus was first reported by Silva et al. [1]. This disease is a new concept among endometrial malignancies. Differentiated carcinoma of the uterine corpus is rare, comprising only $9 \%$ of endometrial cancers, and it is not discoverable until it is at an advanced stage [2].

This carcinoma develops in a polyp-like shape in the uterine body lumen and is characterized histopathologically by a well-differentiated endometrioid carcinoma region on the surface of the tumor and an undifferentiated carcinoma region in the deep part of the tumor demarcated by a clear border [3].

Some reports have described associations between the prognosis and some gene mutations, such as mismatch repair (MMR) gene mutations, POLE, and SWI/SNF complex [4-6]. However, because dedifferentiated carcinoma has biological heterogeneity, is rare, and has a higher rate of mutation than other high-risk endometrial cancers, a comprehensive search for gene mutations and treatment methods for each case is considered to contribute to improving the prognosis of these patients.

Foundation One ${ }^{\circledR}$ CDx gene profiling can comprehensively identify 324 gene mutations using a next-generation sequencer, as well as evaluate biomarkers, such as microsatellite status and tumor mutation burden (detailed information available at https://www.foundationmedicine.com/genomic-testing/foundation-one-cdx). We report a case wherein Foundation One ${ }^{\circledR}$ CDx gene profiling was used to search for gene mutations and treatments for dedifferentiated carcinoma of the uterine corpus.

\section{Case Presentation}

A 49-year-old woman who complained of irregular genital bleeding was referred to our department for detailed examination and treatment after endometrial histology performed in a gynecological clinic established a diagnosis of grade 1 endometrioid cancer. Her family history included breast cancer in her maternal aunt in her $70 \mathrm{~s}$, liver cancer in her maternal uncle in his $70 \mathrm{~s}$, and colorectal cancer in her maternal cousin in his $50 \mathrm{~s}$.

A gynecological examination revealed a polypoid tumor occupying the uterine lumen. Computed tomography and magnetic resonance imaging showed almost no muscle invasion and no suspicion of lymph node or distant organ metastasis; the preoperative diagnosis was endometrial cancer (endometrioid cancer grade 1) stage IA (shown in Fig. 1). The
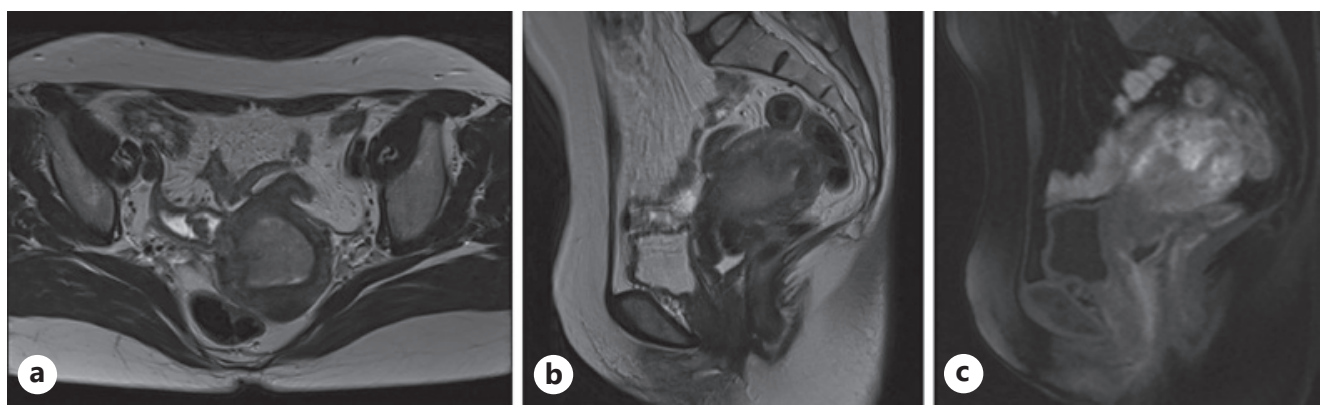

Fig. 1. Images obtained at the first examination; MRI. a, b MRI T2-weighted image. c MRI fat suppression gadolinium contrast T1-weighted image. A polyp-like mass growing in the form of the lumen from the right side of the uterine body. MRI, magnetic resonance imaging. 
Taira et al.: Comprehensive Genomic Profiling for Endometrial Dedifferentiated Carcinoma

carcinosarcoma was differentially diagnosed from the image findings, and transabdominal hysterectomy was performed, which included resection for the para-aortic lymph nodes and omentum.

The postoperative pathological diagnosis was dedifferentiated carcinoma stage IA (pT1aN0M0). Based on previous reports and the presence of undifferentiated carcinoma components, we judged that the risk of recurrence was high, and performed systemic chemotherapy (six courses of paclitaxel and carboplatin therapy) as postoperative adjuvant therapy. The patient was alive without any recurrence 30 months after treatment. Endometrial dedifferentiated carcinoma has a poor prognosis and no established treatment strategy, considering the risk of recurrence; we have searched a treatment strategy by Foundation One ${ }^{\circledR} \mathrm{CDx}$ gene profiling.

\section{Methods}

Immunohistochemical staining, including epithelial membrane antigen, estrogen receptor, progesterone receptor, CD56, synaptophysin, chromogranin A, and CD8, was performed at diagnosis. Analyses of MMR gene-related proteins and immune checkpoint receptors and comprehensive genetic profiling by Foundation One ${ }^{\circledR} \mathrm{CDx}$ gene profiling were performed 26 months after hysterectomy, using surgically removed formalin-fixed paraffinembedded specimens from the uterus, with the informed consent of the patients.

Immunohistochemical staining was evaluated by a pathologist for each of the undifferentiated and well-differentiated regions of dedifferentiated carcinoma. The MMR generelated protein and immune checkpoint receptors were MutL protein homolog 1 (MLH1), post-meiotic segregation increased, S. cerevisiae, 2 (PMS2), MutS protein homolog 2 (MSH2), MutS protein homolog 6 (MSH6), programmed death 1 (PD-1), and programmed deathligand-1 (PD-L1). The uterine corpus with high tumor content was sliced from the formalinfixed paraffin-embedded specimen to a thickness of $5 \mu \mathrm{m}$, and 1 hematoxylin-eosin-stained section and 10 unstained sections were submitted to Foundation One ${ }^{\circledR}$ CDx gene profiling. The tumor content in the specimen was $60 \%$.

The sequence was performed using Illumina HiSeq ${ }^{\circledR} 4000$ (Illumina, San Diego, CA, USA) to identify base substitutions, insertions, and deletions (indels); copy number alternations; and rearrangements. In addition, both the tumor mutational burden and microsatellite instability (MSI) status were assessed. Higher tumor mutational burden was defined as $\geq 16-20$ MutS/Mb, and MSI-High was defined as one mutation in $>30 \%$ of all microsatellite markers. Figure 2 shows the histopathological findings.

In this case, after hematoxylin and eosin staining, we detected grades 1 and 2 endometrioid cancer in the well-differentiated region, and small circular cells with atypia were arranged in a sheet in the undifferentiated region with a clear border. Carcinosarcoma and mixed cancer were excluded because they differed from the patterns on immunohistochemical staining and cell morphology; grade 3 endometrioid carcinoma was also excluded because of its distribution and unclear border from the well-differentiated region. Thus, it was diagnosed as dedifferentiated carcinoma.

In the undifferentiated region, for the epithelial markers, epithelial membrane antigen was partially positive, while estrogen receptor and progesterone receptor were negative. Among the neuroendocrine markers, CD56 was only partially positive, while synaptophysin and chromogranin A were negative (data not shown). These findings are consistent with endometrial dedifferentiated carcinoma.

Regarding MMR gene-related protein, MutL protein homolog 1 was weakly positive in the well-differentiated region and positive in the undifferentiated region, and post-meiotic

\section{Karger'}




\section{Case Reports in Oncology}

\begin{tabular}{l|l}
\hline Case Rep Oncol 2022;15:46-55 \\
\hline DOI: 10.1159/000521897 & $\begin{array}{l}\text { ○ 2022 The Author(s). Published by S. Karger AG, Basel } \\
\text { www.karger.com/cro }\end{array}$ \\
\hline
\end{tabular}

Taira et al.: Comprehensive Genomic Profiling for Endometrial Dedifferentiated Carcinoma
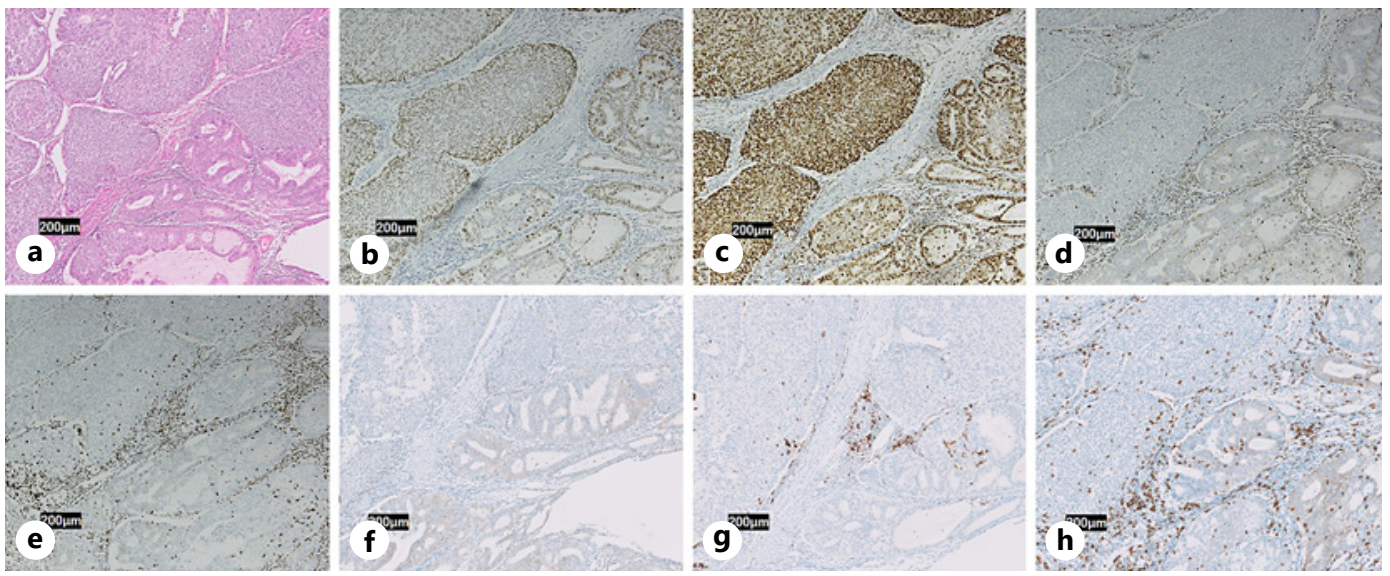

Fig. 2. Pathological findings $(\times 50)$. a Endometrioid cancer grade $1-2$ in the well-differentiated region; small circular cells with atypia are arranged in a sheet in an undifferentiated region with clear border by hematoxylin and eosin staining. b MLH1 was weakly positive in the differentiated carcinoma component and positive in the undifferentiated component. c PMS2 was partially positive in the differentiated carcinoma component and positive in the undifferentiated carcinoma component. $\mathbf{d}$ MSH2 was negative in both components. e MSH6 was weakly positive in both components. $\mathbf{f}$ PD-1 was negative in both components. g PD-L1 was negative in both components. $\mathbf{h}$ CD8 staining reveals an accumulation of cytotoxic T cells in both well-differentiated and undifferentiated regions. MLH1, MutL protein homolog 1; PMS2, post-meiotic segregation increased 2 .

segregation increased 2 was weakly positive in the well-differentiated region and positive in the undifferentiated region. The MSH2 was negative, and the MSH6 was weakly positive in both regions. Both PD-1 and PD-L1 receptors were negative; however, CD8 staining was positive in both regions, suggesting the presence of cytotoxic T cells.

Foundation One ${ }^{\circledR} \mathrm{CDx}$ showed that the microsatellite status was MSI-High and the tumor mutation burden was $44 \mathrm{MutS} / \mathrm{Mb}$ as biomarker findings. Table 1 shows the genomic findings. In addition to mutations in ARID1A, PIK3CA and PTEN mutations were found in MSH6 and MSH2 in the MMR gene. Foundation One ${ }^{\circledR} \mathrm{CDx}$ identified 13 therapies with clinical benefit and 40 clinical trials for genetic mutations and biomarkers in this case.

\section{Discussion/Conclusion}

Dedifferentiated endometrial carcinoma is a rare endometrial malignancy that is biologically heterogeneous in The Cancer Genome Atlas (TCGA) molecular subgroups and reportedly has a higher rate of mutation than other high-risk endometrial cancers $[7,8]$. The gene mutations identified in endometrial dedifferentiated carcinoma by using polymerase chain reaction methods, such as Sanger sequencing were POLE, PIK3CA, PTEN, and the SWI/SNF complex [4-6].

To the best of our knowledge, this is the first report of comprehensive genomic profiling by Foundation One ${ }^{\circledR}$ CDx gene profiling for endometrial dedifferentiated carcinoma. Numerous gene mutations, including the MMR gene, were identified in this case.

MSH2 and MSH6 belong to the MMR gene family and encode DNA MMR proteins and MutS homolog six proteins. Immunohistochemical staining detected a deficiency in the expression of MSH2 protein and MSH6 protein. However, since the MSH2 protein forms a complex (MutS) with the MSH6 protein or the MSH3 protein and functions as a dimer, the findings of immunohistochemical staining, in this case, suggest a mutation in the MSH2 gene. 
Table 1. Genomic mutation findings

Taira et al.: Comprehensive Genomic Profiling for Endometrial Dedifferentiated Carcinoma

\begin{tabular}{ll}
\hline Gene & Alteration \\
\hline ARID1A & A134fs*98 \\
CIC & P1899fs*2 \\
CSF1R & P509fs*14 \\
EGFR & R399* \\
GNA13 & R677H \\
MLL2 & R166* \\
MSH2 & Q791fs*3 \\
MSH3 & P648fs*2 \\
MSH6 & M141fs*6 \\
NF1 & K383fs*32 \\
PBRM1 & F1088f*5 \\
PIK3CA & N78fs*7 \\
PIK3R1 & I279fs*4 \\
PTEN & R108H \\
& G376R \\
SPEN & E256fs*41 \\
\hline
\end{tabular}

Therefore, it is considered that mutations in the MSH6 gene, which are difficult to detect only by immunohistochemical staining, could be detected accurately.

As observed in this case, MSH2 alternations that might result in loss or disruption of the EXO1 interaction, MutS core, or nucleotide triphosphate hydrolase domain are predicted to be inactivating $[9,10]$. MSH6 alternations that result in disruption or loss of the PWWP and/ or ATPase domain are predicted to cause loss of function [11-13].

MSH2 inactivation and MSH6 loss or inactivation are associated with MSI and increased mutation burden [14]. MSI is a condition of genetic hypermutability that generates excessive amounts of short insertion/deletion mutations in the genome; it generally occurs at the microsatellite DNA sequences [15]. MSI-High status indicates a high-level deficiency in MMR and typically correlates with loss of expression of at least one MMR family protein and often two MMR family proteins $[16,17]$.

While approximately $80 \%$ of MSI-High tumors develop owing to somatic inactivation of an MMR pathway protein, approximately $20 \%$ occur owing to germline mutations in one of the MMR genes, which are associated with a condition known as Lynch syndrome [18].

The proportion of deficient MMR (dMMR), which is a surrogate marker of MSI-High, is high in endometrial dedifferentiated carcinoma $[4,19]$. According to a report of TCGA molecular subgroups classification for endometrial dedifferentiated carcinoma and undifferentiated carcinoma, 44\% are MSI (hypermutated) subgroups, which are high compared to the proportion of MSI (hypermutated) subgroups (28\%) in all endometrial cancers [4]. This case is considered to be an MSI (hypermutated) subgroup in the TCGA molecular subgroups classification. Some literature has reported an association between endometrial dedifferentiation carcinoma and dMMR and Lynch syndrome [20, 21]; diagnosis of endometrial dedifferentiated carcinoma is very important because endometrial carcinoma can be a sentinel cancer of Lynch syndrome.

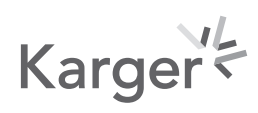


The family history in this case did not meet the Amsterdam Criteria II or revised Bethesda Criteria used for screening for Lynch syndrome [22, 23]. Of all endometrial cancer patients, 2-6\% are diagnosed as having Lynch syndrome, and the probability that patients with endometrial carcinoma who are molecularly evaluated as having suspected Lynch syndrome have a pathogenic gene mutation of Lynch syndrome which is $40-60 \%[24,25]$. In this case, scrutiny of germline MMR gene mutations is considered.

MSI-High and associated increased tumor mutation burden may predict sensitivity to anti-PD-1 and anti-PD-L1 immune checkpoint inhibitors, including the approved therapies with nivolumab, pembrolizumab, atezolizumab, avelumab, and durvalumab [26, 27]. Treatment for endometrial dedifferentiated carcinoma has not yet been established, and Soyama et al. [28] reported that there is no effective adjuvant chemotherapy. Because the Foundation One ${ }^{\circledR}$ CDx gene profiling showed high MSI-High and tumor mutation burden values, pembrolizumab was selected as a therapeutic drug candidate in this case.

Ono et al. [29] reported that dMMR and PD-L1 receptor expression in undifferentiated regions were associated and that a large number of CD8-positive T cells were accumulated, suggested the possibility of improving prognosis by immunotherapy using PD-1/PD-L1 antibodies. However, few reports are describing the actual use of immune checkpoint inhibitors for MSI-High endometrial dedifferentiated carcinoma. Goh et al. [30] reported 15 months of stable disease and the disappearance of ascites using a combined chemotherapy of pembrolizumab and gemcitabine for stage IIIA endometrial dedifferentiated carcinoma that recurred during postoperative paclitaxel and carboplatin therapy.

In our case, immunohistochemical staining detected the accumulation of CD8-positive T cells, but PD-L1 and PD-1 receptors could not be detected. Therefore, a biomarker search by Foundation One ${ }^{\circledR}$ CDx gene profiling suggested the effectiveness of an immune checkpoint inhibitor. In addition, other multiple gene mutations were also reported on targeted therapeutic agents and trials.

PTEN encodes an inositol phosphatase that functions as a tumor suppressor by negatively regulating the phosphatidylinositol 3-kinase (PI3K)-AKT-mTOR pathway; loss of PTEN can lead to uncontrolled cell growth and suppression of apoptosis [31]. PTEN alternations that disrupt the N-terminal PIP2 binding motif, phosphatase domain, C2 domain, C-terminal region, and/or PTEN localization, such as those observed in our case, are predicted to cause loss of function [32-35]. PTEN loss or mutation leads to activation of the PI3K-AKT-mTOR pathway and might predict sensitivity to the inhibitors of this pathway, such as the mTOR inhibitors temsirolimus and everolimus, or the PI3K inhibitor copanlisib in other tumor types [36-38].

PIK3CA encodes p110-alpha, which is the catalytic subunit of PI3K. The PI3K pathway is involved in cell signaling that regulates several critical cellular functions, including cell growth, proliferation, differentiation, motility, and survival $[39,40]$. Clinical and preclinical data in various tumor types indicate that PIK3CA-activating alternations might predict sensitivity to therapies targeting PI3K or AKT $[41,42]$. On the basis of the clinical benefit for patients with PIK3CA mutations and preclinical evidence, PIK3CA-mutated tumors may also respond to $\mathrm{mTOR}$ inhibitors, including everolimus and temsirolimus [43, 44].

ARID1A encodes the AT-rich interactive domain-containing protein $1 \mathrm{~A}$, also known as Baf250a, a member of the SWI/SNF chromatin-remodeling complex. The SWI/SNF complex is one of the chromatin-remodeling factors that transform the chromatin structure by migrating and removing nucleosomes by using energy from ATP hydrolysis. However, the SWI/SNF complex, with ARID1A as a subunit, is reportedly deleted in approximately $70 \%$ of endometrial dedifferentiated carcinoma cases [45]. In recent years, mutations in subunits of the SWI/SNF complex have been detected in various cancers and have been reported to be one of the factors of poor prognosis in endometrial dedifferentiated carcinoma [46]. Mutation, loss, or inactivation of ARID1A has been reported in gynecological cancers, and the gene is

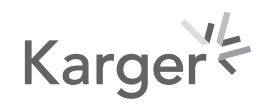


considered a tumor suppressor [47-49]. No treatment has been approved for the mutation or loss of ARID1A in cancer. However, ARID1A-inactivating mutations might lead to sensitivity to ATR inhibitors.

Espinosa et al. [50] evaluated POLE, PIK3CA, PTEN, and ARID1A in 13 cases of endometrial dedifferentiated carcinoma and 8 cases of undifferentiated endometrial carcinoma. They reported that $42 \%$ of the cases with the POLE mutation showed a less advanced stage and had a good prognosis. This is similar to the results found in the POLE-mutant/ultramutated group in the TCGA molecular subgroup classification and thought to be the result of the antitumor effect of cytotoxic T cells [51].

PIK3CA, PTEN, and ARID1A (other than POLE) were not prognostic factors, and these mutations were observed to be overlapping in 70-90\% of the POLE mutations. Comparable findings were observed in the TCGA molecular subgroup classification, which suggests that a threshold possibly exists for the burden of mutations that tumor cells can tolerate. Furthermore, several trials of targeted therapeutic agents for NF1, EGFR, and PIK3R1 gene mutations are underway, and the prognosis is expected to improve in the future.

The Discussion/Conclusion should provide an evaluation of the results. There should be a clear discussion of the implications, significance, and novelty of the research presented and whether the data support or contradict previous studies. In conclusion, the search for genetic mutations and biomarkers by comprehensive genomic profiling may contribute to the establishment of treatments for rare diseases and improve prognosis.

\section{Acknowledgment}

The authors would like to thank Enago (www.enago.jp) for the English language review.

\section{Statement of Ethics}

This study protocol was reviewed and approved by the Ethical Committee of the University of the Ryukyus, approval number 1836. Written informed consent was obtained from the patient for publication of the details of this medical case and any accompanying images.

\section{Conflict of Interest Statement}

Y. Taira declares no conflict of interest. The other authors also declare no conflicts of interest directly relevant to the content of this article.

\section{Funding Sources}

We have no source of funding.

\section{Author Contributions}

Yusuke Taira contributed to project development, data collection and analysis, and manuscript writing. Yuko Shimoji contributed to gynecological management and data collection. Yoshihisa Arakaki contributed to gynecological management and data collection.

\section{Karger'}


Taira et al.: Comprehensive Genomic Profiling for Endometrial Dedifferentiated Carcinoma

Tomoko Nakamoto contributed to gynecological management and data collection. Wataru Kudaka contributed to gynecological managementand data collection. Yoichi Aoki contributed to project development and data analysis.

\section{Data Availability Statement}

All data generated or analyzed during this study are included in this article. Further enquiries can be directed to the corresponding author.

\section{References}

1 Silva EG, Deavers MT, Bodurka DC, Malpica A. Association of low-grade endometrioid carcinoma of the uterus and ovary with undifferentiated carcinoma: a new type of dedifferentiated carcinoma? Int J Gynecol Pathol. 2006;25(1):52-8.

2 Silva EG, Deavers MT, Malpica A. Undifferentiated carcinoma of the endometrium: a review. Pathology. 2007; 39(1):134-8.

3 Kurman RJ, Carcangiu ML, Herrington CS, Young RH. WHO classification of tumours of female reproductive organs. 4th edn. World Health Organization; 2014.

4 Travaglino A, Raffone A, Gencarelli A, Saracinelli S, Riccardi C, Mollo A, et al. Clinico-pathological features associated with mismatch repair deficiency in endometrial undifferentiated/dedifferentiated carcinoma: a systematic review and meta-analysis. Gynecol Oncol. 2021;160(2):579-85.

5 Zhang K, Liu Y, Liu X, Du J, Wang Y, Yang J, et al. Clinicopathological significance of multiple molecular features in undifferentiated and dedifferentiated endometrial carcinomas. Pathology. 2021;53(2):179-86.

6 Kihara A, Amano Y, Matsubara D, Fukushima N, Fujiwara H, Niki T. BRG1, INI1, and ARID1B deficiency in endometrial carcinoma: a clinicopathologic and immunohistochemical analysis of a large series from a single institution. Am J Surg Pathol. 2020;44(12):1712-24.

7 Travaglino A, Raffone A, Mascolo M, Guida M, Insabato L, Zannoni GF, et al. TCGA molecular subgroups in endometrial undifferentiated/dedifferentiated carcinoma. Pathol Oncol Res. 2020;26(3):1411-6.

8 Rosa-Rosa JM, Leskelä S, Cristóbal-Lana E, Santón A, López-García MÁ, Muñoz G, et al. Molecular genetic heterogeneity in undifferentiated endometrial carcinomas. Mod Pathol. 2016;29(11):1390-8.

9 Li GM. Mechanisms and functions of DNA mismatch repair. Cell Res. 2008;18:85-98.

10 Lützen A, de Wind N, Georgijevic D, Nielsen FC, Rasmussen LJ. Functional analysis of HNPCC-related missense mutations in MSH2. Mutat Res. 2008;645:44-55.

11 Li F, Mao G, Tong D, Huang J, Gu L, Yang W, et al. The histone mark H3K36me3 regulates human DNA mismatch repair through its interaction with MutS $\alpha$. Cell. 2013;153:590-600.

12 Warren JJ, Pohlhaus TJ, Changela A, Iyer RR, Modrich PL, Beese LS. Structure of the human MutSalpha DNA lesion recognition complex. Mol Cell. 2007;26:579-92.

13 Geng H, Sakato M, DeRocco V, Yamane K, Du C, Erie DA, et al. Biochemical analysis of the human mismatch repair proteins hMutS $\alpha$ MSH2(G674A)-MSH6 and MSH2-MSH6(T1219D). J Biol Chem. 2012;287:9777-91.

14 McConechy MK, Talhouk A, Li-Chang HH, Leung S, Huntsman DG, Gilks CB, et al. Detection of DNA mismatch repair (MMR) deficiencies by immunohistochemistry can effectively diagnose the microsatellite instability (MSI) phenotype in endometrial carcinomas. Gynecol Oncol. 2015;137:306-10.

15 Kocarnik JM, Shiovitz S, Phipps AI. Molecular phenotypes of colorectal cancer and potential clinical applications. Gastroenterol Rep. 2015;3:269-76.

16 You JF, Buhard O, Ligtenberg MJ, Kets CM, Niessen RC, Hofstra RM, et al. Tumours with loss of MSH6 expression are MSI-H when screened with a pentaplex of five mononucleotide repeats. Br J Cancer. 2010;103:1840-5.

17 Bairwa NK, Saha A, Gochhait S, Pal R, Gupta V, Bamezai RN. Microsatellite instability: an indirect assay to detect defects in the cellular mismatch repair machinery. Methods Mol Biol. 2014;1105:497-509.

18 Lynch HT, Lynch PM, Lanspa SJ, Snyder CL, Lynch JF, Boland CR. Review of the Lynch syndrome: history, molecular genetics, screening, differential diagnosis, and medicolegal ramifications. Clin Genet. 2009;76:1-18.

19 Hacking S, Jin C, Komforti M, Liang S, Nasim M. MMR deficient undifferentiated/dedifferentiated endometrial carcinomas showing significant programmed death ligand-1 expression (sp 142) with potential therapeutic implications. Pathol Res Pract. 2019;215:152552.

20 Tafe LJ, Garg K, Chew I, Tornos C, Soslow RA. Endometrial and ovarian carcinomas with undifferentiated components: clinically aggressive and frequently underrecognized neoplasms. Mod Pathol. 2010;23:781-9.

21 Yokomizo R, Yamada K, Iida Y, Kiyokawa T, Ueda K, Saito M, et al. Dedifferentiated endometrial carcinoma: a report of three cases and review of the literature. Mol Clin Oncol. 2017;7:1008-12.

22 Vasen HF, Watson P, Mecklin JP, Lynch HT. New clinical criteria for hereditary nonpolyposis colorectal cancer (HNPCC, Lynch syndrome) proposed by the International Collaborative Group on HNPCC. Gastroenterology. 1999;116:1453-6.

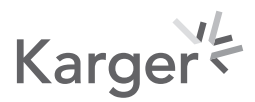


23 Umar A, Boland CR, Terdiman JP, Syngal S, de la Chapelle A, Rüschoff J, et al. Revised Bethesda Guidelines for hereditary nonpolyposis colorectal cancer (Lynch syndrome) and microsatellite instability. J Natl Cancer Inst. 2004;96(4):261-8.

24 Ferguson SE, Aronson M, Pollett A, Eiriksson LR, Oza AM, Gallinger S, et al. Performance characteristics of screening strategies for Lynch syndrome in unselected women with newly diagnosed endometrial cancer who have undergone universal germline mutation testing. Cancer. 2014;120:3932-9.

25 Takahashi K, Sato N, Sugawara T, Kato A, Sato T, Shimizu D, et al. Clinical characteristics of Lynch-like cases collaterally classified by Lynch syndrome identification strategy using universal screening in endometrial cancer. Gynecol Oncol. 2017;147:388-95.

26 Lal N, Beggs AD, Willcox BE, Middleton GW. An immunogenomic stratification of colorectal cancer: implications for development of targeted immunotherapy. Oncoimmunology. 2015;4:e976052.

27 Gatalica Z, Snyder C, Maney T, Ghazalpour A, Holterman DA, Xiao N, et al. Programmed cell death 1 (PD-1) and its ligand (PD-L1) in common cancers and their correlation with molecular cancer type. Cancer Epidemiol Biomarkers Prev. 2014;23:2965-70.

28 Soyama H, Takano M, Miyamoto M, Kato M, Goto T, Furuya K. Dedifferentiated endometrioid adenocarcinoma of the uterus: a case report. Eur J Gynaecol Oncol. 2016;37:426-9.

29 Ono R, Nakayama K, Nakamura K, Yamashita H, Ishibashi T, Ishikawa M, et al. Dedifferentiated endometrial carcinoma could be a target for immune checkpoint inhibitors (anti PD-1/PD-L1 antibodies). Int J Mol Sci. 2019;20:3744.

30 Goh C, Farah BL, Ho WY, Wong SL, Goh CHR, Chew SH, et al. Dedifferentiated endometrioid adenocarcinoma of the uterus: a case series and review of literature. Gynecol Oncol Rep. 2020;32:100538.

31 Simpson L, Parsons R. PTEN: life as a tumor suppressor. Exp Cell Res. 2001;264:29-41.

32 Campbell RB, Liu F, Ross AH. Allosteric activation of PTEN phosphatase by phosphatidylinositol 4,5-bisphosphate. J Biol Chem. 2003;278:33617-20.

33 Rodríguez-Escudero I, Oliver MD, Andrés-Pons A, Molina M, Cid VJ, Pulido R. A comprehensive functional analysis of PTEN mutations: implications in tumor- and autism-related syndromes. Hum Mol Genet. 2011;20: 4132-42.

34 Lee JO, Yang H, Georgescu MM, Di Cristofano A, Maehama T, Shi Y, et al. Crystal structure of the PTEN tumor suppressor: implications for its phosphoinositide phosphatase activity and membrane association. Cell. 1999; 99:323-34.

35 Valiente M, Andrés-Pons A, Gomar B, Torres J, Gil A, Tapparel C, et al. Binding of PTEN to specific PDZ domains contributes to PTEN protein stability and phosphorylation by microtubule-associated serine/threonine kinases. J Biol Chem. 2005;280:28936-43.

36 Nguyen HN, Yang JM Jr, Rahdar M, Keniry M, Swaney KF, Parsons R, et al. A new class of cancer-associated PTEN mutations defined by membrane translocation defects. Oncogene. 2015;34:3737-43.

37 Patnaik A, Appleman LJ, Tolcher AW, Papadopoulos KP, Beeram M, Rasco DW, et al. First-in-human phase I study of copanlisib (BAY 80-6946), an intravenous pan-class I phosphatidylinositol 3-kinase inhibitor, in patients with advanced solid tumors and non-Hodgkin's lymphomas. Ann Oncol. 2016;27:1928-40.

38 Courtney KD, Corcoran RB, Engelman JA. The PI3K pathway as drug target in human cancer. J Clin Oncol. 2010; 28:1075-83

39 Dreyling M, Morschhauser F, Bouabdallah K, Bron D, Cunningham D, Assouline SE, et al. Phase II study of copanlisib, a PI3K inhibitor, in relapsed or refractory, indolent or aggressive lymphoma. Ann Oncol. 2017;28: 2169-78.

40 Samuels Y, Diaz LA Jr, Schmidt-Kittler O, Cummins JM, Delong L, Cheong I, et al. Mutant PIK3CA promotes cell growth and invasion of human cancer cells. Cancer Cell. 2005;7:561-73.

41 Engelman JA. Targeting PI3K signalling in cancer: opportunities, challenges and limitations. Nat Rev Cancer. 2009;9:550-62.

42 André F, Ciruelos E, Rubovszky G, Campone M, Loibl S, Rugo HS, et al. Alpelisib for PIK3CA-mutated, hormone receptor-positive advanced breast cancer. N Engl J Med. 2019;380:1929-40.

43 Fritsch C, Huang A, Chatenay-Rivauday C, Schnell C, Reddy A, Liu M, et al. Characterization of the novel and specific PI3K $\alpha$ inhibitor NVP-BYL719 and development of the patient stratification strategy for clinical trials. Mol Cancer Ther. 2014;13:1117-29.

44 Park HS, Lim SM, Kim S, Kim S, Kim HR, Kwack K, et al. Pilot study of a next-generation sequencing-based targeted anticancer therapy in refractory solid tumors at a Korean Institution. PLoS One. 2016;11: e0154133.

45 Janku F, Tsimberidou AM, Garrido-Laguna I, Wang X, Luthra R, Hong DS, et al. PIK3CA mutations in patients with advanced cancers treated with PI3K/AKT/mTOR axis inhibitors. Mol Cancer Ther. 2011;10: 558-65.

46 Karnezis AN, Hoang LN, Coatham M, Ravn S, Almadani N, Tessier-Cloutier B, et al. Loss of switch/sucrose nonfermenting complex protein expression is associated with dedifferentiation in endometrial carcinomas. Mod Pathol. 2016;29:302-14.

47 Guan B, Wang TL, Shih I-M. ARID1A, a factor that promotes formation of SWI/SNF-mediated chromatin remodeling, is a tumor suppressor in gynecologic cancers. Cancer Res. 2011;71:6718-27.

48 Wiegand KC, Shah SP, Al-Agha OM, Zhao Y, Tse K, Zeng T, et al. ARID1A mutations in endometriosis-associated ovarian carcinomas. N Engl J Med. 2010;363:1532-43. 
49 Jones S, Wang TL, Shih I-M, Mao TL, Nakayama K, Roden R, et al. Frequent mutations of chromatin remodeling gene ARID1A in ovarian clear cell carcinoma. Science. 2010;330(6001):228-31.

50 Espinosa I, Lee CH, D’Angelo E, Palacios J, Prat J. Undifferentiated and dedifferentiated endometrial carcinomas with POLE exonuclease domain mutations have a favorable prognosis. Am J Surg Pathol. 2017;41: 1121-8.

51 Cancer Genome Atlas Research Network; Kandoth C, Schultz N, Cherniack AD, Akbani R, Liu Y, et al. Integrated genomic characterization of endometrial carcinoma. Nature. 2013;497:67-73. 